\title{
The development of a eustress sensing system using In-Ear EEG
}

\author{
Kazutaka Ueda, Yasuhiko Imamura, and Takuya Ibaraki
}

\begin{abstract}
It is necessary to remain in a eustress state while concentrating on a task to increase productivity. This study aimed to develop a eustress sensing system using In-Ear electroencephalograph (EEG). We demonstrated that EEG data measured by In-Ear EEG could be used to predict a subjective eustress state while performing eustress-inducing tasks.
\end{abstract}

\section{INTRODUCTION}

It is necessary to concentrate and immerse oneself in a task (e.g., a flow state) to increase productivity. Hans Selye categorized stress during a situation into distress (negative state) and eustress (positive state). We have developed an In-Ear electroencephalograph (EEG) as a simple method for sensing the eustress state such as flow state. Herein, we measured the EEG during eustress and distress tasks using the In-Ear EEG, and verified the validity of eustress sensing.

\section{METHODS}

We recruited six healthy men $(22.8 \pm 0.8$ years $)$ for the experiment. This study was approved by the Research Ethics Committee of the University of Tokyo (approval number: KE21-1). We used tasks to induce either eustress or distress state separately based on an N-back task [1]. In the eustress task, the participants were mentioned that they would receive a monetary reward and a "right" sound for each correct answer in the 2-back task. In contrast, in the distress task, they were mentioned obtaining a monetary punishment and a buzzer sound for each incorrect answer in the 3-back task. Following each task, they responded to the flow state scale for occupational tasks (Yoshida et al., 2013) as an indicator of eustress. We recorded the EEG during tasks from the left and right ear canals with an In-Ear EEG (VIE ZONE, VIE STYLE Inc., reference electrode: the back of neck, Fig.1 left). We also recorded the scalp EEG using an EEG amplifier system (Brain Amp DC, Brain Products $\mathrm{GmbH}$ ) with passive electrodes according to the international 10-20 system (Fp1/2, F3/4, C3/4, P3/4, O1/2, F7/8, T7/8, P7/8, Fz, Cz, and Pz, average reference). This was compared with the EEG measured by the In-Ear EEG. EEG data was analyzed using MATLAB and EEGLAB (Delorme \& Makeig, 2004).

\section{RESULTS}

In the eustress task, the flow state scale scores were significantly higher than that in the distress task. Our findings confirmed that eustress was induced in this task.

K. Ueda is with the Department of Mechanical Engineering, Graduate School of Engineering, The University of Tokyo, Tokyo, Japan.

(Email: ueda@hnl.t.u-tokyo.ac.jp)
To examine the signal homology between the EEG obtained by the In-Ear EEG and scalp EEG, we segmented each EEG data every $4 \mathrm{~s}$, and performed a spectral analysis. Moreover a correlation analysis was performed on the time course of the EEG power at $1 \mathrm{~Hz}$ intervals from $3-30 \mathrm{~Hz}$. Fig. 1 depicts the mean values of correlation coefficients for all frequency bands and participants for each electrode position of the In-Ear EEG (left and right ear) and Scalp EEG. The correlation between the In-Ear EEG and ipsilateral scalp electrode tended to be high. In particular, there were high correlations between the left electrode of the In-Ear EEG and the left lateral, parietal, and central scalp electrodes.

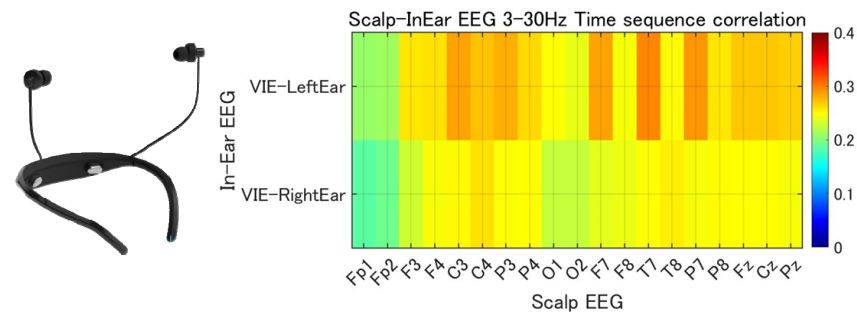

Figure 1. Overview of the In-Ear EEG "VIE ZONE" (left), Correlation coefficient of time series of EEG power of Scalp EEG and In-Ear EEG (right)

We constructed a least absolute shrinkage and selection operator model to predict the eustress state from the EEG data obtained by the In-Ear EEG. The correlation coefficient evaluated the prediction accuracy. The flow state scale scores obtained for each task were used as supervisory data. In contrast, the power of each electrode $(3-30 \mathrm{~Hz})$, the difference in power between the left and right electrodes, and the power of the difference in amplitude between left and right in the EEG data were used to predict the eustress state. Moreover, data from the EAR2BRAIN model (a technique to reconstruct the other scalp electrode signals from the in-ear EEG signals, a concept similar to electrical fingerprint (Keynan et al., 2019)) were also constructed for each participant, and used as predictors. The prediction accuracy using the EAR2brain model $(r=.80 \pm .07)$ was significantly higher than that using only In-Ear EEG data $(r=.77 \pm .08)$.

\section{DISCUSSION \& CONCLUSION}

Our findings indicate the possibility of predicting the eustress state from EEG data using In-Ear EEG. Considering the advantage of the wearable device, the evaluation technique of eustress state and neuro-feedback technique using In-Ear EEG will likely be introduced in actual workplace.

\section{REFERENCES}

[1] N. Tateyama, K. Ueda, \& M. Nakao, "Development of an active sensing system for distress detection using skin conductance response," IEEE 8th International Conference on Affective Computing and Intelligent Interaction (ACII), Cambridge, United Kingdom, 2019, pp.353-358. https://doi.org/10.1109/ACII.2019.8925442 